\title{
WILEY-VCH
}

DOI: 10.1002/ ((please add manuscript number))

Article type: Full Paper

\section{Tuning the Luminescence of Tin Oxide Low Dimensional Structures in the Near Infrared Range by in-situ doping during a Vapor-Solid Growth Process}

Miguel García-Tecedor ${ }^{1}$, Félix del Prado ${ }^{1}$, Dorcas Torres ${ }^{2}$, David Maestre ${ }^{1}$ and Ana Cremades $^{1 *}$

Dedicated to Professor Javier Piqueras

${ }^{1}$ Departamento Física de Materiales, Facultad de Físicas, Universidad Complutense de Madrid, Madrid 28040, Spain

${ }^{2}$ Natural Science and Mathematics Department, Inter American University, Bayamon, Puerto

Rico

*E-mail: cremades@fis.ucm.es

Keywords: vapor-solid, tin oxide, cathodoluminescence

\begin{abstract}
Tin oxide low dimensional structures increasingly attract attention due to their wide application area. Indeed, by attaining new morphologies and properties the potential applications might increase the device portfolio. Furthermore, an adequate combination of doped $\mathrm{SnO}_{2}$ nano- and microstructures could enable multi-functionality and totally new applications. The latter might be the case of low dimensional tin oxide structures emitting in the near infrared range, which is below the energy of the common visible luminescence of tin oxide. The ability to obtain near infrared luminescence from tin oxide is tested by doping insitu during a vapor-solid growth using $\mathrm{Li}, \mathrm{Cu}$ and $\mathrm{Cr}$ containing precursors in the initial mixture with tin oxide or metallic tin powders. Luminescence around $1.5 \mathrm{eV}$ is obtained for all the samples with morphologies varying from microtubes to rods and belts depending on the specific dopant and the Sn-based precursor.
\end{abstract}

\section{Introduction}

Tin oxide is a wide band gap material with $\mathrm{E}_{\mathrm{g}}$ around 3,6 eV which presents applications in gas sensors, ${ }^{[1]}$ solar cells, ${ }^{[2]}$ catalysis, ${ }^{[3]}$ batteries $^{[4]}$ or as a diluted magnetic semiconductor (DMSO), ${ }^{[5]}$ among others. Many of these applications can be improved by reducing the size of tin oxide and by adequate doping. The controlled doping of nanostructures is a difficult task in which great efforts are invested, as it is of high relevance for the control of the performance of $\mathrm{SnO}_{2}$-based devices. Tin nanostructures such as nanoparticles, belts, plates 


\section{WILEY-VCH}

and wires have been successfully doped with different elements such as the transition metal chromium, ${ }^{[6-7]}$ vanadium, ${ }^{[8-9]}$ and manganese. ${ }^{[10-11]}$ Other elements such as rare earths are also commonly used to dope tin oxide, being erbium a paradigmatic example. ${ }^{[12-13]}$ Less attention has been paid so far to other doping elements such as copper. ${ }^{[14]}$ The interest in doping tin oxide with copper is usually based on its behaviour as an oxidative catalyst for hydrocarbons to achieve a considerable degree of sensitivity and selectivity, improving the response to $\mathrm{H}_{2} \mathrm{~S}$ in gas sensors. ${ }^{[15]}$ Moreover, co-doping with different elements can lead to overcome limitations of single-doped $\mathrm{SnO}_{2}$ and widen the applicability of $\mathrm{SnO}_{2} \cdot{ }^{[16-17]}$

Recently, other low dimensional structures of transparent conducting oxides (TCO) such as micro and nanotubes are attracting increasing attention due to their improved performances in energy storage, among others. ${ }^{[18]}$ Its fabrication by template-based methods, ${ }^{[19]}$ gives rise to polycrystalline and mesoporous, instead of highly crystalline materials. On the contrary, the TCO tubes grown by an analogous vapor-solid method used in this work, ${ }^{[20-24]}$ are single crystalline and do not need the use of a catalyst. Doping with different elements efficiently influences the optical properties of TCO tubular structures, which can be used for different applications, for example, as a luminescence material for the visible range or as optical resonator combined with wave-guiding behaviour. ${ }^{[23,25]}$

In this work, we have achieved the doping of $\mathrm{SnO}_{2}$ elongated nano and microstructures with $\mathrm{Cu}$, as well as the codoping with $\mathrm{Li}$ and $\mathrm{Cr}$ by a vapor-solid method. The influence of the Snbased precursor material and the doping in the size, shape and morphology of the as-grown structures is demonstrated, as well as the ability to shift the luminescence of tin oxide into the near infrared region, for which the best results are obtained for $\mathrm{Li}$ and Cr codoped tubular microstructures. The luminescence emission of tin oxide is commonly reported as a wide emission in the visible range ${ }^{[26]}$ comprising several complex bands related to intrinsic defects, mainly oxygen vacancies. Therefore, the ability to tune the luminescence of tin oxide tubes in 


\section{WILEY-VCH}

the NIR could be useful in sensors and biochemical detection, ${ }^{[27]}$ widening the applicability of tin oxide.

\section{Results and discussion}

Undoped tin oxide low dimensional structures have been achieved starting from $\mathrm{SnO}_{2}$ powders at $1400^{\circ} \mathrm{C}$, by a vapor solid growth as reported elsewhere. ${ }^{[21]}$ The preferential morphologies obtained by this method are microtubes, with lateral (110) surfaces and $<001>$ growth directions, as determined by electron backscatter diffraction (EBSD) and Raman polarized measurements. ${ }^{[28-29]}$ The growth mechanism is based on the anisotropy of the rutile tin oxide structure for which the most stable surface is the $\{110\} .{ }^{[23]}$ These microtubes can be doped during growth with $\mathrm{Li}$ or $\mathrm{Cr}$ by incorporating the convenient element in the powder precursors, while maintaining the tube morphology with slight changes. ${ }^{[28-29]}$ In the case of codoping with $\mathrm{Li}$ and $\mathrm{Cr}$, the surface of the pellet is fully covered by microstructures after the thermal treatments performed at $1400{ }^{\circ} \mathrm{C}$ during $10 \mathrm{~h}$, as it can be observed in Figure 1a. The majority of these structures are microtubes, although there is a small amount of other morphologies such as microrods and microplates. The amount of microtubes in the $\mathrm{Cr}-\mathrm{Li}$ codoped samples is higher than in the undoped $\mathrm{SnO}_{2}$ ones. In Figure 1(b-c) some of these tubular microstructures can be observed with higher magnification. The microtubes show square or rectangular cross-sections with widths between 50 and $200 \mu \mathrm{m}$ and lengths up to 5 $\mathrm{mm}$. The co-doping process with $\mathrm{Cr}$ and $\mathrm{Li}$ in $\mathrm{SnO}_{2}$ introduces some morphological changes in the structures as compared to undoped material. First of all, the length of the as-grown microstructures was increased with the $\mathrm{Cr}-\mathrm{Li}$ codoping, as compared with undoped or separately Li or Cr doped tubes. 


\section{WILEY-VCH}
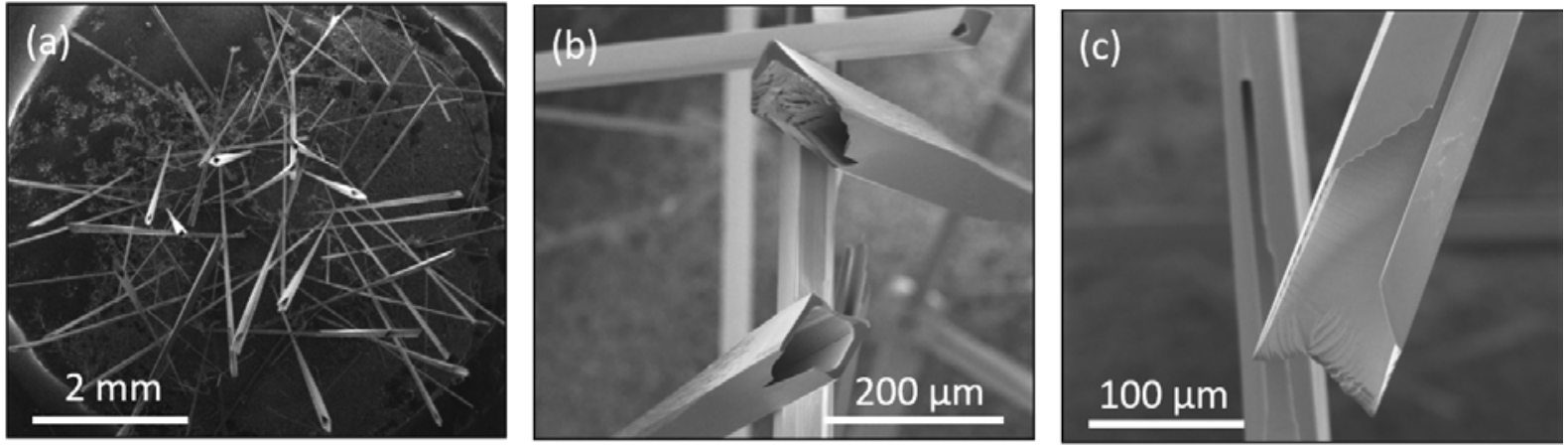

Figure 1. (a) SEM image of the aspect of the $\mathrm{SnO}_{2}$ : $\mathrm{Cr}, \mathrm{Li}$ pellet after the thermal treatment. (b), (c) and (d) SEM images showing different codoped $\mathrm{SnO}_{2}$ :Cr,Li microtubes.

The co-doped samples seem to have simultaneously the morphological influence of both $\mathrm{Cr}$ and Li dopants. To get a better understanding of this fact, in Figure 2, a comparison of undoped and differently doped $\mathrm{SnO}_{2}$ microtubes is shown.
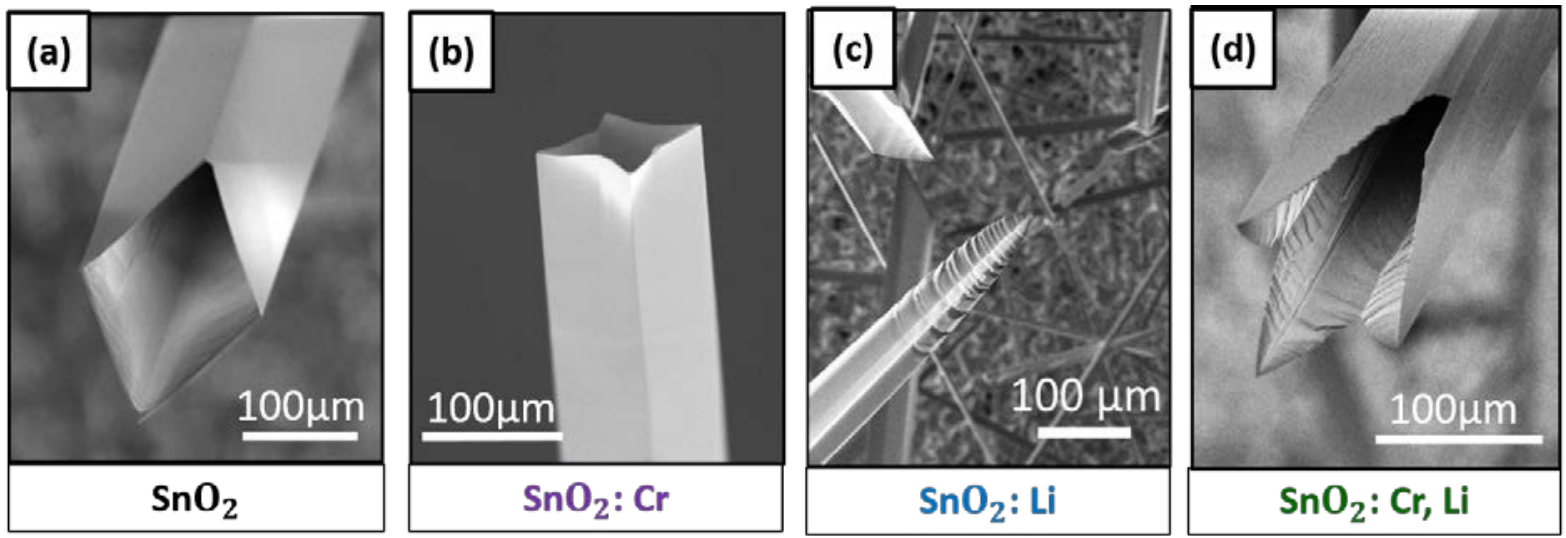

Figure 2. SEM images of (a) undoped, (b) Cr doped, (c) Li doped and (d) Cr and Li co-doped $\mathrm{SnO}_{2}$ microtubes.

As it can be observed in Figure 2, different SEM images show the evolution of the morphological aspect of the as-grown microtubes by the introduction of different dopants. In Figure 2(a) an undoped $\mathrm{SnO}_{2}$ microtube can be observed with square cross-section, no angular ending and smooth inner and outer surfaces. In Figure 2(b), a Cr doped structure is shown where a characteristic angular four tip-ending can be observed. These four tip-ending microtubes were also observed in $\mathrm{Cr}$ doped rutile $\mathrm{TiO}_{2}$ microtubes. ${ }^{[20]}$ In Figure 2(c) Li doped 


\section{WILEY-VCH}

$\mathrm{SnO}_{2}$ microstructures are observed, where a snake tail-ending can be observed as it was previously reported. ${ }^{[28]}$ Finally, in Figure 2(d), the aspect of a Cr and Li co-doped microtube is shown, where a mixture of the four-tip (three in that case because the fourth tip is missing in this structure) and the snake tail ending can be observed.

In order to study the crystalline structure of the as-grown microtubes some of them were carefully detached from the pellet surface and analyzed by XRD. Figure 3(a) shows the XRD measurements acquired in both undoped and $\mathrm{Li}$ and $\mathrm{Cr}$ codoped microtubes for comparison. XRD measurements confirm that the codoped $\mathrm{SnO}_{2}$ :Cr, $\mathrm{Li}$ microtubes consist of cassiterite $\mathrm{SnO}_{2}$ with tetragonal rutile structure, as the undoped ones. No presence of any ternary compounds or any rest of precursor powders has been detected by this technique. The preferential crystalline orientations belong to the family planes $\{110\}$, in agreement with the results for undoped, $\mathrm{Cr}$ doped and $\mathrm{Li}$ doped $\mathrm{SnO}_{2}$ microtubes. ${ }^{[28-29]}$ Therefore, the doping process does not vary the growth direction and the family of planes, which form the lateral surfaces of the microtubes.
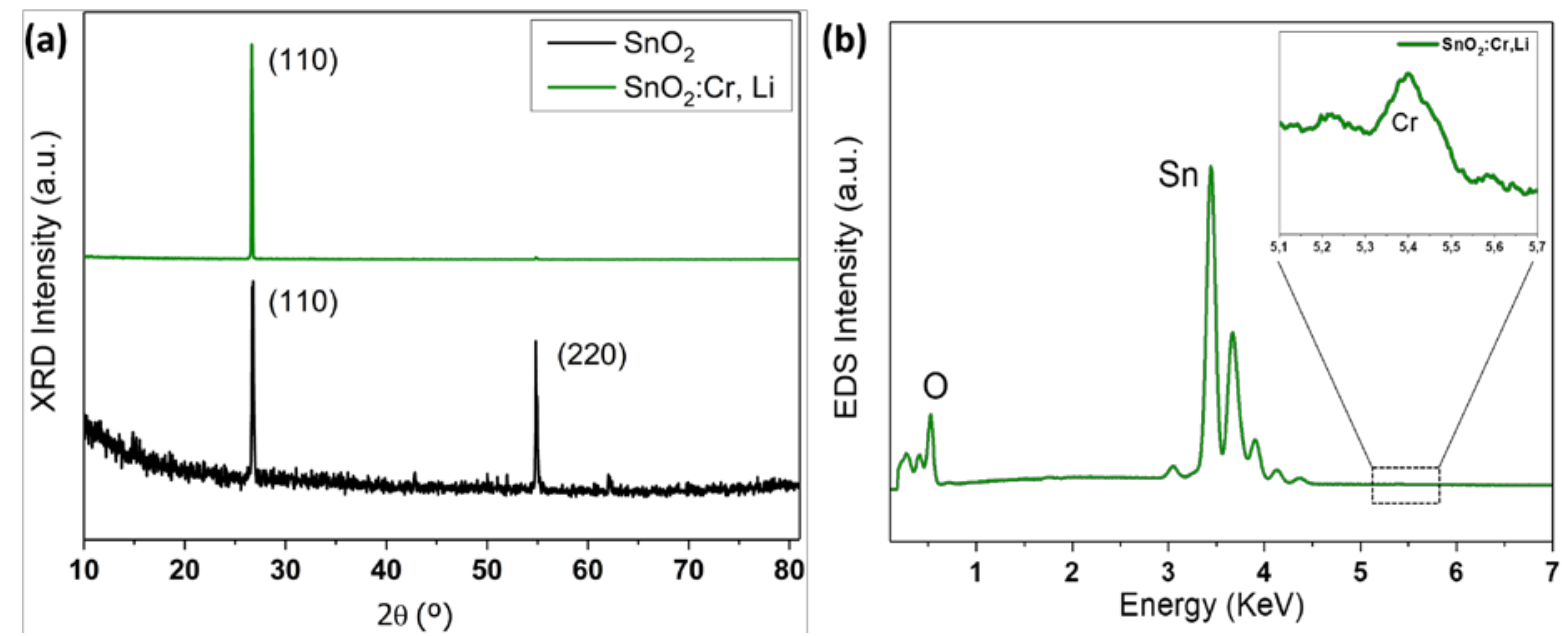

Figure 3. XRD patterns acquired on undoped and codoped $\mathrm{SnO}_{2}$ : $\mathrm{Cr}$, Li samples and (b) EDS spectrum from the codoped $\mathrm{SnO}_{2}$ : $\mathrm{Cr}$,Li sample. 


\section{WILEY-VCH}

The chemical composition of the microtubes was also analyzed in terms of EDS measurements. The EDS spectrum acquired in the codoped $\mathrm{SnO}_{2}: \mathrm{Cr}, \mathrm{Li}$ sample is shown in Figure 3(b). Peaks corresponding to Sn and O can be clearly observed. The inset in Figure 4 shows an enlarged region of the spectrum where a weak peak corresponding to $\mathrm{Cr} \mathrm{K}_{\alpha}$ lines can be observed, confirming the presence of $\mathrm{Cr}$ in the analyzed microtubes. The amount of $\mathrm{Cr}$ incorporated in these microstructures is below $1 \%$ at, as estimated from the quantification of the corresponding EDS spectrum. Moreover, $\mathrm{Cr}$ is homogeneously distributed along the microtubes according to EDS measurements and in agreement with previous works. ${ }^{[7,29]}$ On the other hand, as lithium is a light element it cannot be detected by the EDS technique. However, due to the observed morphology modifications similarly to the reference $\mathrm{SnO}_{2}: \mathrm{Li}$ samples, the Li incorporation in the codoped microstructures is assumed to be achieved as previously demonstrated by different techniques ${ }^{[28]}$ for the reference $\mathrm{SnO}_{2}$ : $\mathrm{Li}$ tubes. The achievement of simultaneous incorporation of $\mathrm{Cr}$ and Li codopants is also supported by the variation of the luminescence behavior with respect to the reference samples, as explained in detail below.

The temperature window for achieving the growth of elongated microstructures of tin oxide depends on the selected precursor. The growth temperature ranges between 1300 and $1400{ }^{\circ} \mathrm{C}$ when using tin oxide as precursor. In order to lower the growth temperature to around $600{ }^{\circ} \mathrm{C}$ other precursors could be used as metallic tin, ${ }^{[30-31]}$ which also changes the morphology and reduces the size of the obtained structures giving raise mainly to the growth of nanowires. Alternatively, microstructures could be obtained at intermediate temperatures around $900^{\circ} \mathrm{C}$ by adding to metallic tin powders a high amount of metallic copper. In this case, copper is introduced as a dopant into the tin oxide rutile structure and the final morphology are microrods and belts, as shown in Figure 4(a) and 4(b) for a Cu:Sn ratio of 2:1. The highest 


\section{WILEY-VCH}

copper content is obtained in the belts and quantified around 3 cationic \% by EDS (see EDS Sn and $\mathrm{Cu}$ mappings in Figure $4(\mathrm{c}-\mathrm{d})$ ). The $\mathrm{Cu}$ doped $\mathrm{SnO}_{2}$ microbelts have been characterized by XRD, as it is shown in Figure 4(e), which can be indexed with the tin oxide rutile structure, as it can be in the shown in the spectrum. Ternary compounds, intermediate phases or metallic $\mathrm{Cu}$ or $\mathrm{Sn}$ from the precursors have been discarded, as an indicative of an adequate diffusion of $\mathrm{Cu}$ atoms into the $\mathrm{SnO}_{2}$ lattice during the thermal treatment, in good agreement with the homogeneous distribution of $\mathrm{Sn}$ and $\mathrm{Cu}$ along the grown microbelts observed in the EDS mappings.
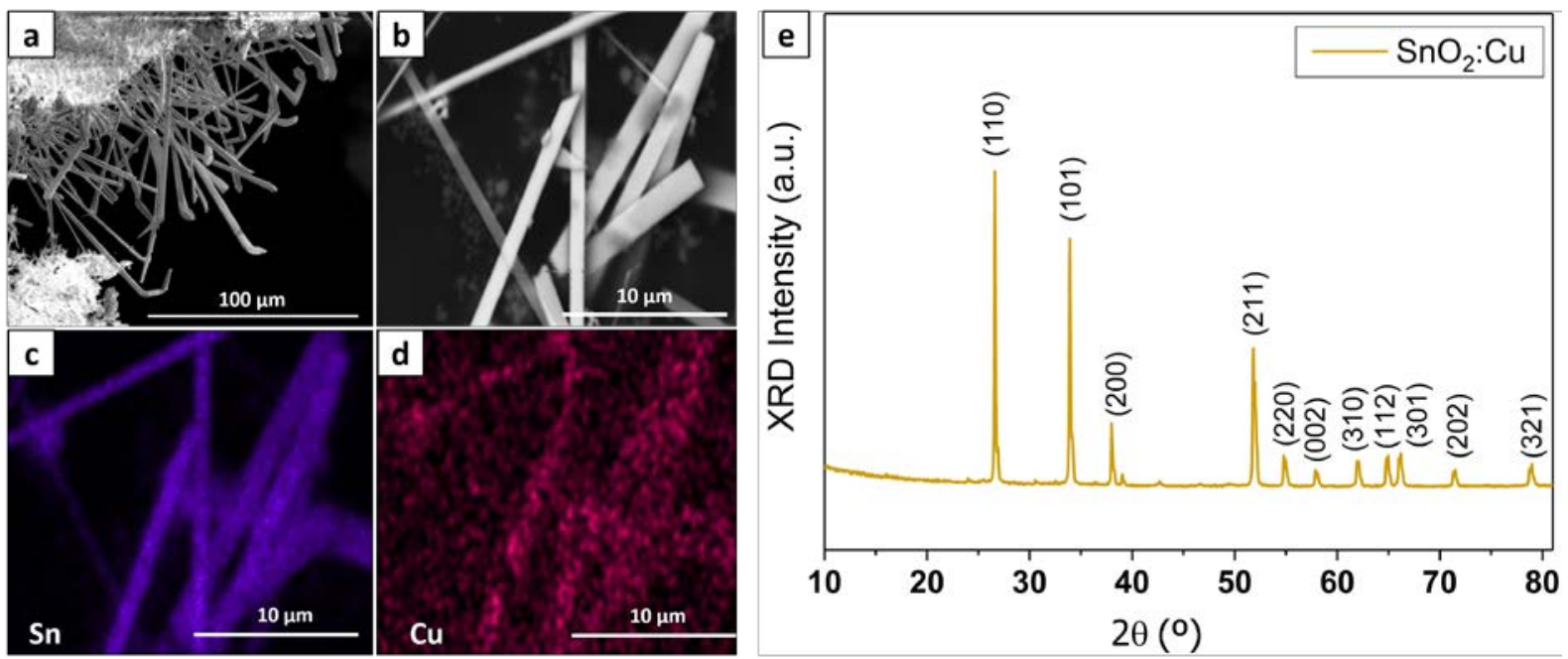

Figure 4. SEM images of the microstructures present in the $\mathrm{Cu}$ doped sample: (a) microrods and (b) belts, (c)-(d) EDS mapping of elemental Sn and Cu corresponding to the belts of (b), respectively and (e) XRD pattern of $\mathrm{Cu}$ doped $\mathrm{SnO}_{2}$ samples.

In order to achieve a better understanding of our system, the CL spectra at low temperature of the $\mathrm{Cu}$ doped and $\mathrm{Li}-\mathrm{Cr}$ codoped $\mathrm{SnO}_{2}$ microstructures are shown for comparison with the spectra of the reference materials in Figure 5(a). 


\section{WILEY-VCH}
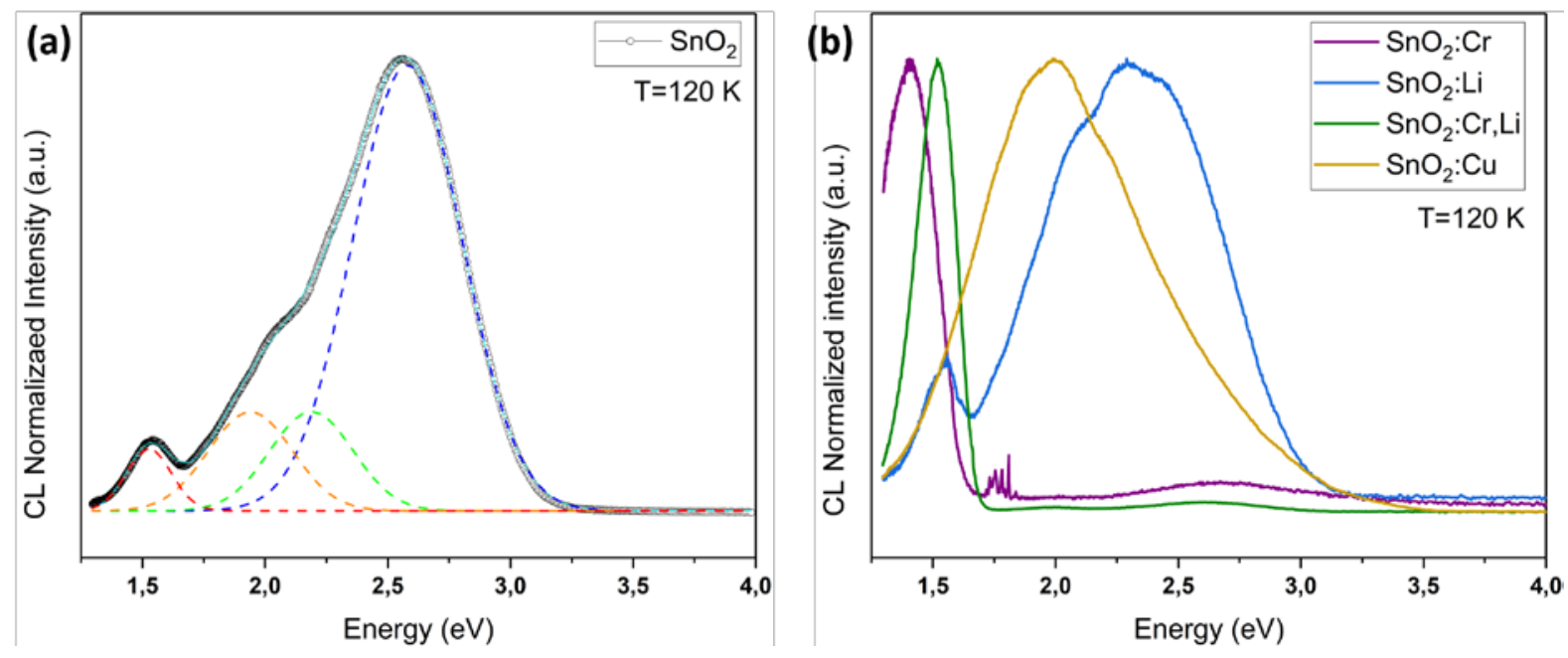

Figure 5. (a) CL spectra acquired on the undoped microtubes, (b) Cr doped, Li doped $\mathrm{Cu}$ doped and codoped $\mathrm{SnO}_{2}: \mathrm{Cr}, \mathrm{Li}$ samples.

As observed in Figure 5(a), the CL spectrum of undoped tubes $\mathrm{SnO}_{2}$ consists of a wide visible emission with four different contributions centered at 1.5, 1.98, 2.25 and $2.58 \mathrm{eV}$, respectively. The first emission at $1.5 \mathrm{eV}$ presents a reduced intensity and is scarcely reported for undoped tin oxide material. Some authors ${ }^{[32]}$ have attributed this NIR band to a high surface density of defects states, which is in agreement with the high surface/volume ratio that exhibit the microstructures describe in this work. The orange $(1.98 \mathrm{eV})$ and the green emission $(2.25 \mathrm{eV})$ are associated with oxygen vacancies ${ }^{[21]}$ and complex defects involving oxygen vacancies, ${ }^{[21]}$ while the blue emission $(2.58 \mathrm{eV})$, is related to surface states. ${ }^{[21,33]}$

The incorporation of Li modifies the CL response by promoting the emission around $1.5 \mathrm{eV}$, as explained by F. del Prado et al. ${ }^{[28]}$ On the other hand, no sharp lines were observed in the $\mathrm{SnO}_{2}: \mathrm{Li}$ spectrum at about $1.70 \mathrm{eV}(729.32 \mathrm{~nm})$ corresponding to ${ }^{2} \mathrm{~S}-{ }^{2} \mathrm{P}_{0}$ intraionic transitions due to $\mathrm{Li}^{+}$in other TCO's such as $\mathrm{Ga}_{2} \mathrm{O}_{3}$ or $\mathrm{TiO}_{2}$ as reported by some authors. ${ }^{[34-35]}$ An allowed transition, ${ }^{2} \mathrm{P}_{0}{ }^{-2} \mathrm{~S}$ attributed to $\mathrm{Li}^{+}$cations, ${ }^{[36]}$ centered at $1.52 \mathrm{eV}(815.8 \mathrm{~nm})$ has also been reported. However, in our case the broad NIR emission at $1.52 \mathrm{eV}$ is detected not only in the $\mathrm{Li}$ doped microstructures but also in the undoped ones, as shown in Figure 5(a). Therefore, an origin related to $\mathrm{Li}$ intraionic emissions can be disregarded in this case. A 


\section{WILEY-VCH}

similar radiative recombination observed in $\mathrm{Cu}$, $\mathrm{Co}$ and $\mathrm{Fe}$ doped $\mathrm{SnO}_{2}$ films have been attributed by Korotcenkov et al. ${ }^{[37]}$ to Sn interstitials. Analogously, in our samples a band at around $1.65 \mathrm{eV}$ is induced by $\mathrm{Cu}$ doping, although with reduced relative intensity, as compared to the Li doping samples. The state of charge of copper incorporated in the tin oxide rutile structure is unknown. Preferential incorporation of copper as $\mathrm{Cu}^{2+}$ could be presumed, as this ion is more stable than the $\mathrm{Cu}^{+}$ion, although further direct measurements are needed to properly asses this point as well as the probability to find $\mathrm{Cu}$ ions in both states of charge. For both samples, $\mathrm{Li}$ and $\mathrm{Cu}$ doped ones, the intrinsic visible bands of tin oxide are the main contribution to the luminescence of the structures, although the relative intensity of the different visible bands is not the same after the introduction of $\mathrm{Li}$ or $\mathrm{Cu}$. Doping $\mathrm{SnO}_{2}$ with $\mathrm{Cr}$ introduces two main differences in the $\mathrm{SnO}_{2} \mathrm{CL}$ emission spectra. The first one is the emission centered at $1.79 \mathrm{eV}$ associated with intraionic transitions of $\mathrm{Cr}^{3+}$ in octahedral positions. ${ }^{[38]}$ The second one is the new emission located around $1.4 \mathrm{eV}$ associated with intrinsic defects present in $\mathrm{SnO}_{2}$ and promoted by doping. ${ }^{[29]}$ In the $\mathrm{SnO}_{2}$ :Cr doped samples, a small contribution from the blue $\mathrm{SnO}_{2}$ emission still remains.

The CL spectrum of the codoped sample $\mathrm{SnO}_{2}$ :Cr, $\mathrm{Li}$ consists of a narrow and intense emission in the near-IR, centered at about $1.5 \mathrm{eV}(\sim 825 \mathrm{~nm})$, and a weak emission centered at around $2.7 \mathrm{eV}(\sim 477 \mathrm{~nm})$. The luminescence has been successfully shifted to the near infrared for the codoped samples, which could turn in new applications of tin oxide as for example in bio-imaging. The NIR luminescence of the Cr-Li codoped $\mathrm{SnO}_{2}$ samples have been analyzed in more detail. The CL spectra acquired in the sample at different temperatures ranging from 120-300 K can be observed in Figure 6, where it can be appreciated a clear increase in the total CL signal as the temperature decreases. 
WILEY-VCH
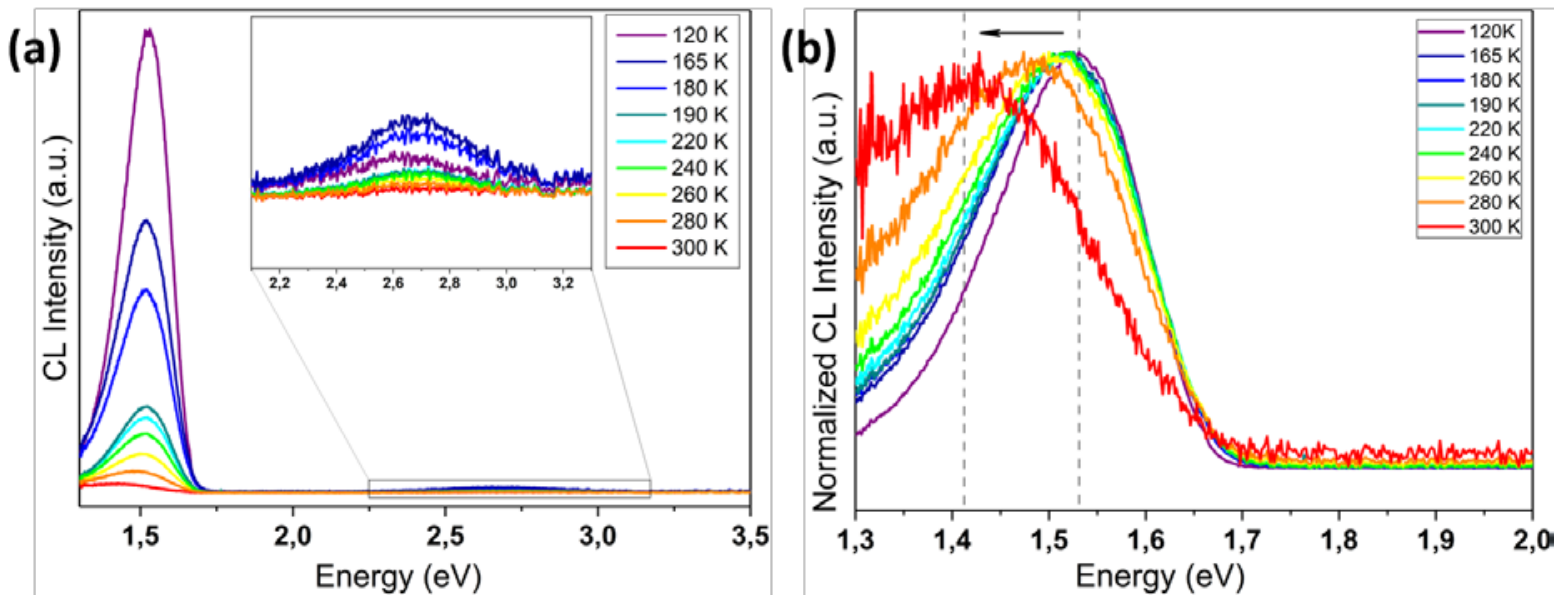

Figure 6. (a) CL spectrum acquired on a microtube of a codoped $\mathrm{SnO}_{2}$ : $\mathrm{Cr}$, $\mathrm{Li}$ sample at different temperatures, with an inset showing and enlargement of the visible emission. (b) Normalized CL spectra of the NIR region.

At the inset in Figure 8(a) an enlargement of CL visible emission is shown. The main band centered at around $2.7 \mathrm{eV}$ is totally quenched as the temperature increases up to $300 \mathrm{~K}$. According to the normalized CL spectra shown in Figure 8(b), the maximum in the emission of the $\mathrm{SnO}_{2}$ :Cr,Li samples appears to be centered at $1.55 \mathrm{eV}$ at low temperatures and a slight shift towards $1.42 \mathrm{eV}$ is observed as temperature increases up to $300 \mathrm{~K}$. This result could indicate that this emission is a complex band composed by the contributions at 1.4 and $1.6 \mathrm{eV}$ from $\mathrm{Cr}$ and $\mathrm{Li}$ ion respectively which evolve differently with temperature. However, the observed behavior with temperature is also characteristic of radiative transitions involving charge transfer, ${ }^{[39]}$ and other complex defects involving $\mathrm{Li}$ and $\mathrm{Cr}$ ions cannot be disregarded. Furthermore, Li codoping is a common strategy to enhance efficiency in phosphors ${ }^{[40]}$ and several mechanisms could contribute to this effect, among others charge compensation as $\mathrm{Li}^{+}$ can compensate the cationic vacancies produced by the incorporation of trivalent or divalent ions to phosphor materials and eventually promote more ions incorporating into the host lattice.

In summary, the observed luminescence of doped samples should be tentatively related to the effects of the incorporation of dopants into the rutile lattice of tin oxide, which could induce 


\section{WILEY-VCH}

the generation of a complex defect to compensate the charge imbalance due to the substitution of tin by $\mathrm{Li}, \mathrm{Cu}$, or $\mathrm{Cr}$, respectively. The final peak position seems to be related to the specific dopant element, as for $\mathrm{Cr}$ doped samples the NIR emission is centered at around $1.4 \mathrm{eV}$, for $\mathrm{Li}$ and $\mathrm{Cu}$ doping it moves towards higher energies around $1.6 \mathrm{eV}$, meanwhile for $\mathrm{Li}-\mathrm{Cr}$ codoped samples its position is around $1.5 \mathrm{eV}$.

\section{Conclusion}

Doping tin oxide during a vapor-solid growth using $\mathrm{Li}, \mathrm{Cu}$ and $\mathrm{Cr}$ containing precursors in the initial mixture enables the achievement of near infrared luminescence from tin oxide low dimensional structures. The best results are achieved by codoping tin oxide with $\mathrm{Li}$ and $\mathrm{Cr}$, obtaining single crystalline microtubes by a vapor-solid growth at $1400{ }^{\circ} \mathrm{C}$. The tubes exhibit an intense luminescence at around $1.5 \mathrm{eV}$ with simultaneous quenching of the visible emission band of tin oxide. Unravelling the origin of this luminescence band needs further research, although our results suggest its relation to intrinsic tin oxide defects generated to compensate charge imbalance due to the incorporation of the doping elements into the tin oxide lattice. This band seems to be composed by several contributions depending on the charge state of the doping element. Although copper doping does not promote the quenching of the visible band, it also allows a NIR emission around $1.6 \mathrm{eV}$, meanwhile the morphology variability increases, obtaining rods and belts at lower treatment temperatures.

\section{Experimental Section}

$\mathrm{SnO}_{2}$ nano and microstructures were fabricated following a vapor-solid mechanism by thermal treatments of $\mathrm{SnO}_{2}$ powders under controlled Ar flow. Doping with $\mathrm{Cu}$ or codoping with $\mathrm{Li}$ and $\mathrm{Cr}$ was achieved during the growth by selecting a convenient mixture of the following commercial powders as starting materials: $\mathrm{SnO}_{2}$ (Sigma-Aldrich, 99.9\%), metallic Sn (Sigma-Aldrich, 99\%), metallic Cu (Sigma-Aldrich, 99 \%), $\mathrm{Li}_{2} \mathrm{CO}_{3}$ (Labkem, 99.0\%) and $\mathrm{Cr}_{2} \mathrm{O}_{3}$ (Sigma-Aldrich, 99.9\%). After several experiments, a 5 \% weight concentration of Li 


\section{WILEY-VCH}

and $\mathrm{Cr}$ in the initial mixture with $\mathrm{SnO}_{2}$ powders was chosen for the growth of $\mathrm{Li}$-Cr codoped samples, meanwhile for $\mathrm{Cu}$ doping the starting mixture contained a weight ratio of metallic $\mathrm{Cu}$ to $\mathrm{Sn}$ of 2:1. The obtained samples are named hereinafter, $\mathrm{SnO}_{2}: \mathrm{Cu}$, and $\mathrm{SnO}_{2}: \mathrm{Cr}, \mathrm{Li}$ for doped and codoped samples, respectively. Undoped samples and samples doped with only $\mathrm{Cr}$ or $\mathrm{Li}$ are also used in this work as reference materials, and will be named as $\mathrm{SnO}_{2}, \mathrm{SnO}_{2}$ : $\mathrm{Cr}$, and $\mathrm{SnO}_{2}: \mathrm{Li}$, respectively. The fabrication and characterization of the reference materials have been reported elsewhere. ${ }^{[22,28-29]}$

In order to obtain the precursor material, a milling process was carried out in order to homogenize the corresponding powder mixture, by using a mechanical agate ball mill (in a Restch S100) during 5 hours at $150 \mathrm{rpm}$. The homogenized powders were pressed into a pellet (7 mm diameter, $3 \mathrm{~mm}$ high) using a MEGA KP-30th mechanical press. Afterwards, the pellets were introduced in a furnace under an argon flow. The thermal treatments were carried out during 10 hours in a MUFLA RHF Carbolite 1500 with an Ar flow of $1.5 \mathrm{~L} / \mathrm{min}$ at temperatures between 900-1400 ${ }^{\circ} \mathrm{C}$. Specifically, for the undoped $\mathrm{SnO}_{2}, \mathrm{SnO}_{2}: \mathrm{Cr}$ and $\mathrm{SnO}_{2}: \mathrm{Cr}$,Li samples the optimized temperature was $1400{ }^{\circ} \mathrm{C}$, whereas for the sample $\mathrm{SnO}_{2}: \mathrm{Li}$ the temperature employed was $1350^{\circ} \mathrm{C}$ and for $\mathrm{Cu}$ doping the temperature was $900{ }^{\circ} \mathrm{C}$.

The obtained microstructures were studied by X-Ray Diffraction (XRD) using a Panalytical X'Pert Pro Alpha1 diffractometer with the working radiation of $\mathrm{Cu}\left(\mathrm{K}_{\alpha}\right)(\lambda=1.5418 \AA)$. The morphology of the as grown samples was studied by scanning electron microscopy (SEM) in a Leica 440 Stereoscan and a FEI-Inspect $\mathrm{S}$ at accelerating voltages of between $15-20 \mathrm{kV}$. The luminescence of the samples was also studied at variable temperature by cathodoluminescence (CL) in a Hitachi S-2500. Compositional analysis was performed by energy-dispersive x-ray spectroscopy in a SEM, using an XFlash 4010 detector.

\section{Acknowledgements}

The work was supported by MINECO/FEDER projects: MAT 2016-81720-REDC and MAT 2015-65274-R. The authors are grateful to Prof. Javier Piqueras (Universidad Complutense de Madrid) for being an inspiring reference in their research careers. 


\section{WILEY-VCH}

Received: ((will be filled in by the editorial staff))

Revised: ((will be filled in by the editorial staff)) Published online: ((will be filled in by the editorial staff))

\section{References}

[1] Zhang, Y.; Kolmakov, A.; Chretien, S.; Metiu, H.; Moskovits, M., Control of catalytic reactions at the surface of a metal oxide nanowire by manipulating electron density inside it. Nano Letters 2004, 4 (3), 403-407.

[2] Li, Z. D.; Zhou, Y.; Yu, T.; Liu, J. G.; Zou, Z. G., Unique Zn-doped $\mathrm{SnO}_{2}$ nanoechinus with excellent electron transport and light harvesting properties as photoanode materials for high performance dye-sensitized solar cell. Crystengcomm 2012, 14 (20), 64626468.

[3] Wang, W. W.; Zhu, Y. J.; Yang, L. X., ZnO-SnO2 hollow spheres and hierarchical nanosheets: Hydrothermal preparation, formation mechanism, and photocatalytic properties. Advanced Functional Materials 2007, 17 (1), 59-64.

[4] Kim, C.; Noh, M.; Choi, M.; Cho, J.; Park, B., Critical size of a nano $\mathrm{SnO}_{2}$ electrode for Li-secondary battery. Chemistry of Materials 2005, 17 (12), 3297-3301.

[5] Archer, P. I.; Radovanovic, P. V.; Heald, S. M.; Gamelin, D. R., Low-temperature activation and deactivation of high-Curie-temperature ferromagnetism in a new diluted magnetic semiconductor: $\mathrm{Ni}^{2+}$-Doped $\mathrm{SnO}_{2}$. J Am Chem Soc 2005, 127 (41), 14479-87.

[6] Zhang, L.; Ge, S.; Zuo, Y.; Wang, J.; Qi, J., Ferromagnetic properties in undoped and Cr-doped $\mathrm{SnO}_{2}$ nanowires. Scripta Materialia 2010, 63 (10), 953-956.

[7] Garcia-Tecedor, M.; Maestre, D.; Cremades, A.; Piqueras, J., Influence of Cr Doping on the Morphology and Luminescence of $\mathrm{SnO}_{2}$ Nanostructures. Journal of Physical Chemistry C 2016, 120 (38), 22028-22034.

[8] Wang, C. T.; Chen, M. T.; Lai, D. L., Vanadium - Tin Oxide Nanoparticles with Gas - Sensing and Catalytic Activity. Journal of the American Ceramic Society 2011, 94 (12), 4471-4477.

[9] Peche-Herrero, M. A.; Maestre, D.; Ramirez-Castellanos, J.; Cremades, A.; Piqueras, J.; Gonzalez-Calbet, J. M., The controlled transition-metal doping of $\mathrm{SnO}_{2}$ nanoparticles with tunable luminescence. Crystengcomm 2014, 16 (14), 2969-2976.

[10] Herrera, M.; Maestre, D.; Cremades, A.; Piqueras, J., Growth and Characterization of Mn Doped $\mathrm{SnO}_{2}$ Nanowires, Nanobelts, and Microplates. The Journal of Physical Chemistry C 2013, 117 (17), 8997-9003.

[11] Chi, J.; Ge, H.; Wang, J.; Zuo, Y.; Zhang, L., Synthesis and electrical and magnetic properties of Mn-doped SnO2 nanowires. Journal of Applied Physics 2011, 110 (8), 083907.

[12] Wu, J.; Coffer, J. L., Strongly emissive erbium-doped tin oxide nanofibers derived from sol gel/electrospinning methods. The Journal of Physical Chemistry C 2007, 111 (44), 16088-16091. 


\section{WILEY-VCH}

[13] del-Castillo, J.; Rodríguez, V.; Yanes, A.; Méndez-Ramos, J., Energy transfer from the host to $\mathrm{Er}^{3+}$ dopants in semiconductor $\mathrm{SnO}_{2}$ nanocrystals segregated in sol-gel silica glasses. Journal of Nanoparticle Research 2008, 10 (3), 499-506.

[14] Santilli, C. V.; Pulcinelli, S. H.; Brito, G.; Briois, V., Sintering and crystallite growth of nanocrystalline copper doped tin oxide. The Journal of Physical Chemistry B 1999, 103 (14), 2660-2667.

[15] Niranjan, R.; Patil, K.; Sainkar, S.; Mulla, I., High $\mathrm{H}_{2} \mathrm{~S}$-sensitive copper-doped tin oxide thin film. Materials chemistry and physics 2003, 80 (1), 250-256.

[16] Liu, S.-J.; Liu, C.; Juang, J.; Fang, H., Room-temperature ferromagnetism in Zn and Mn codoped $\mathrm{SnO}_{2}$ films. Journal of Applied Physics 2009, 105 (1), 013928.

[17] Nomura, K.; Okabayashi, J.; Okamura, K.; Yamada, Y., Magnetic properties of Fe and Co codoped $\mathrm{SnO}_{2}$ prepared by sol-gel method. Journal of Applied Physics 2011, 110 (8), 083901.

[18] Eustache, E.; Tilmant, P.; Morgenroth, L.; Roussel, P.; Patriarche, G.; Troadec, D.; Rolland, N.; Brousse, T.; Lethien, C., Silicon - Microtube Scaffold Decorated with Anatase $\mathrm{TiO}_{2}$ as a Negative Electrode for a 3D Litium - Ion Microbattery. Advanced Energy Materials 2014, 4 (8).

[19] Bae, C.; Yoo, H.; Kim, S.; Lee, K.; Kim, J.; Sung, M. M.; Shin, H., Template-directed synthesis of oxide nanotubes: fabrication, characterization, and applications. Chemistry of Materials 2008, 20 (3), 756-767.

[20] Vasquez, G. C.; Peche-Herrero, M. A.; Maestre, D.; Cremades, A.; RamirezCastellanos, J.; Gonzalez-Calbet, J. M.; Piqueras, J., Cr doped titania microtubes and microrods synthesized by a vapor-solid method. Crystengcomm 2013, 15 (27), 5490-5495.

[21] Maestre, D.; Cremades, A.; Piqueras, J., Growth and luminescence properties of micro- and nanotubes in sintered tin oxide. Journal of Applied Physics 2005, 97 (4), 044316.

[22] Maestre, D.; Cremades, A.; Piqueras, J.; Gregoratti, L., Thermal growth and structural and optical characterization of indium tin oxide nanopyramids, nanoislands, and tubes. Journal of Applied Physics 2008, 103 (9), 093531.

[23] Garcia-Tecedor, M.; del Prado, F.; Bueno, C.; Vasquez, G. C.; Bartolome, J.; Maestre, D.; Diaz, T.; Cremades, A.; Piqueras, J., Tubular micro- and nanostructures of TCO materials grown by a vapor-solid method. Aims Materials Science 2016, 3 (2), 434-447.

[24] Maestre, D.; Hernández, E.; Cremades, A.; Amati, M.; Piqueras, J., Synthesis and Characterization of Small Dimensional Structures of Er-Doped $\mathrm{SnO}_{2}$ and Erbium-Tin-Oxide. Crystal Growth \& Design 2012, 12 (5), 2478-2484.

[25] García-Tecedor, M.; Maestre, D.; Cremades, A.; Piqueras, J., Tailoring optical resonant cavity modes in $\mathrm{SnO}_{2}$ microstructures through doping and shape engineering. Journal of Physics D: Applied Physics 2017, 50 (41), 415104.

[26] Maestre, D.; Cremades, A.; Piqueras, J., Cathodoluminescence of defects in sintered tin oxide. Journal of Applied Physics 2004, 95 (6), 3027-3030.

[27] Smith, E. J.; Schulze, S.; Kiravittaya, S.; Mei, Y.; Sanchez, S.; Schmidt, O. G., Lab-ina-tube: detection of individual mouse cells for analysis in flexible split-wall microtube resonator sensors. Nano letters 2010, 11 (10), 4037-4042.

[28] del Prado, F.; Cremades, A.; Ramírez-Castellanos, J.; Maestre, D.; González-Calbet, J. M.; Piqueras, J., Effect of lithium doping and precursors on the microstructural, surface 


\section{WILEY-VCH}

electronic and luminescence properties of single crystalline microtubular tin oxide structures. CrystEngComm 2017, 19 (30), 4321-4329.

[29] Garcia-Tecedor, M.; Maestre, D.; Cremades, A.; Piqueras, J., Growth and characterization of $\mathrm{Cr}$ doped $\mathrm{SnO}_{2}$ microtubes with resonant cavity modes. Journal of Materials Chemistry C 2016, 4 (24), 5709-5716.

[30] Duan, J.; Yang, S.; Liu, H.; Gong, J.; Huang, H.; Zhao, X.; Zhang, R.; Du, Y., Single crystal $\mathrm{SnO}_{2}$ zigzag nanobelts. Journal of the American Chemical Society 2005, 127 (17), 6180-6181.

[31] Junhong, D.; Jiangfeng, G.; Hongbo, H.; Xiaoning, Z.; Guangxu, C.; Zhong-Zhen, Y.; Shaoguang, Y., Multiform structures of $\mathrm{SnO}_{2}$ nanobelts. Nanotechnology 2007, 18 (5), 055607.

[32] Wang, D. N.; Yang, J. L.; Li, X. F.; Wang, J. J.; Li, R. Y.; Cai, M.; Sham, T. K.; Sun, X. L., Observation of Surface/Defect States of $\mathrm{SnO}_{2}$ Nanowires on Different Substrates from X-ray Excited Optical Luminescence. Crystal Growth \& Design 2012, 12 (1), 397-402.

[33] Fillard, J. P.; Demurcia, M., Low-Temperature Photoluminescence in $\mathrm{SnO}_{2}$ HighResistivity Monocrystals. Physica Status Solidi a-Applied Research 1975, 30 (1), 279-287.

[34] Lopez, I.; Alonso-Orts, M.; Nogales, E.; Mendez, B.; Piqueras, J., Influence of Li doping on the morphology and luminescence of $\mathrm{Ga}_{2} \mathrm{O}_{3}$ microrods grown by a vapor-solid method. Semiconductor Science and Technology 2016, 31 (11), 115003.

[35] Kallel, W.; Bouattour, S.; Ferreira, L. V.; do Rego, A. B., Synthesis, XPS and luminescence (investigations) of $\mathrm{Li}^{+}$and/or $\mathrm{Y}^{3+}$ doped nanosized titanium oxide. Materials Chemistry and Physics 2009, 114 (1), 304-308.

[36] Wiese, W. L.; Fuhr, J. R., Accurate Atomic Transition Probabilities for Hydrogen, Helium, and Lithium. Journal of Physical and Chemical Reference Data 2009, 38 (3), 565720.

[37] Korotcenkov, G.; Cho, B. K.; Nazarov, M.; Noh, D. Y.; Kolesnikova, E. V., Cathodoluminescence studies of un-doped and $(\mathrm{Cu}, \mathrm{Fe}$, and $\mathrm{Co}$ )-doped tin dioxide films deposited by spray pyrolysis. Current Applied Physics 2010, 10 (4), 1123-1131.

[38] Nogales Díaz, E.; García, J. A.; Méndez Martín, B.; Piqueras de Noriega, J., Red luminescence of $\mathrm{Cr}$ in $\mathrm{B}_{-}-\mathrm{Ga}_{2} \mathrm{O}_{3}$ nanowires. Journal of applied physics 2007, 101 (3).

[39] Ye, S.; Song, E. H.; Zhang, Q. Y., Transition Metal - Involved Photon Upconversion. Advanced Science 2016, 3 (12).

[40] Chen, J.; Li, C.; Hui, Z.; Liu, Y., Mechanisms of $\mathrm{Li}^{+}$ions in the emission enhancement of $\mathrm{KMg}_{4}\left(\mathrm{PO}_{4}\right)_{3}: \mathrm{Eu}^{2+}$ for white light emitting diodes. Inorganic chemistry 2017, 56 (3), 11441151. 


\section{WILEY-VCH}

\section{Table of Contents (ToC)}

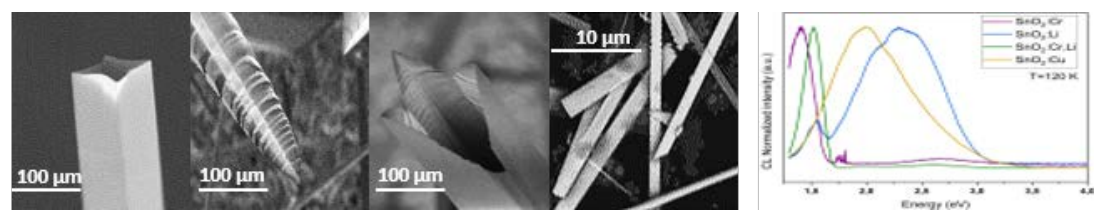

Doping tin oxide with $\mathrm{Li}, \mathrm{Cu}$ and $\mathrm{Cr}$ enables the achievement of near infrared luminescence from tin oxide microstructures. In the present work, different doped low dimensional structures have been obtained by a vapor-solid method. Luminescence around 1.5 $\mathrm{eV}$ is obtained for all the samples with morphologies varying from microtubes to belts depending on the specific dopant and the Sn-based precursor. 